\title{
Human kinesin superfamily member 4 is dominantly localized in the nuclear matrix and is associated with chromosomes during mitosis
}

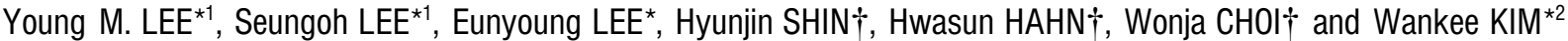 \\ *Institute for Medical Sciences, School of Medicine, Ajou University, Suwon 442-749, South Korea, and †Department of Biological Sciences, \\ College of Natural Sciences, Ewha Women's University, Seoul 120-750, South Korea
}

In a previous study, we identified the human counterpart of murine kinesin superfamily member 4 (KIF4), a microtubulebased motor protein [Oh, Hahn, Torrey, Shin, Choi, Lee, Morse and Kim (2000) Biochim. Biophys. Acta 1493, 219-224]. As an initial step to understand the function(s) of human KIF4, its subcellular localization in HeLa cells was examined by using immunocytochemical and subcellular fractionation methods, and it was found that most KIF4 is localized in the nucleus. Since murine KIF4 is known to transport cytoplasmic vesicles, dominant nuclear localization of the human counterpart was somewhat surprising. Subsequent subnuclear fractionation revealed predominant association of KIF4 with the nuclear matrix. These results clearly indicate that human KIF4 is, at least, a nuclear protein. In further confirmation of this conclusion, the hexapeptide PKLRRR (amino acids 773-778) in the molecule was found to function as a nuclear localization signal. During the mitotic phase of the cell cycle, human KIF4 was associated with the chromosomes, suggesting that human KIF4 might be a microtubule-based mitotic motor, with DNA as its cargo.

Key words: chromosomal association, KIF4 motor protein, nuclear localization signal.

\section{INTRODUCTION}

Microtubule-dependent movement is fundamental to many biological processes, including cell division [1,2] and intracellular transport of membranous organelles in higher eukaryotic cells $[1,3]$. The force for the movement is derived from ATP hydrolysis by microtubule-based mechanochemical motor proteins: the cytoplasmic dyneins and the kinesin superfamily (KIF) of proteins or kinesin-like proteins (KLPs) [4,5]. During interphase in many cell types, the microtubules are radially polarized with the minus end (slow growing) localized in the vicinity of the nucleus and the plus end (fast growing) orientated towards the cell membrane. The movement of the motor proteins along the microtubules is unidirectional; in general, most kinesins move cargoes outwards toward the cytoplamic membrane, whereas cytoplasmic dyneins move cargoes inwards toward the nucleus.

Conventional kinesin was first identified in squid giant axon $[6,7]$ and chick brain [8], and consists of two heavy chains and two light chains $[9,10]$. The kinesin heavy chain (KHC) is composed of three domains: an N-terminal globular motor domain, which contains the microtubule-binding site, and the ATPase consensus sequence, which generates force for movement on microtubules at the expense of ATP hydrolysis [11]; a central $\alpha$-helical coiled-coil stalk domain, responsible for dimerization with other molecules; and a C-terminal binding domain, which interacts with the light chains and possibly with cargoes [12,13]. A number of genes encoding proteins closely related to KHC have been identified in several organisms [14], and all of the kinesin-related proteins encoded by these genes have domains similar to the motor domain of KHC. In contrast, no significant sequence similarity was detected for the stalk and binding domains. The diversity of the KIF suggests that each KIF member may have a specific function, even though many of the functions are not well defined.
Molecular biological approaches unravelled a large number of KIF members, including more than 20 identified in the mouse [15]. Of these, murine KIF4 was isolated from mouse brains and was found to be an anterograde motor protein for transporting membranous organelles in juvenile neurons and other cells $[16,17]$. Recent studies [18,19] showing that murine KIF4 proteins were possibly associated with those of various retroviruses, including HIV type 1, led to the identification of a human counterpart of murine KIF4 [20]. Human KIF4 cDNA consists of a $60 \mathrm{bp} \mathrm{5^{ \prime }}$ untranslated region (UTR), a $3696 \mathrm{bp}$ open reading frame (ORF) and a $740 \mathrm{bp}$ complete $3^{\prime}$ UTR, and is predicted to encode a $140 \mathrm{kDa}$ protein of 1232 amino acids. Although overall identity of human KIF4 with murine KIF4 is $82 \%$ and $85 \%$ at the nucleic acid and amino acid levels respectively, the function(s) of human KIF4 remains obscure.

Scrutiny of the human KIF4 ORF reveals several clusters of basic amino acid residues. Some of them possess three to five positively charged amino acids within a hexapeptide sequence, which is a characteristic of a simple nuclear localization signal (NLS). These sequences immediately prompted us to examine the possibility that human KIF4 might be a nuclear protein and have a different function(s) other than the transport of membranous organelles. Indeed, we report in the present study that human KIF4 is a nuclear protein dominantly associated with the nuclear matrix at interphase and with chromosomes during mitosis, suggesting an as yet unidentified function of human KIF4.

\section{EXPERIMENTAL}

\section{Cell cultures}

HeLa (human cervix epitheloid carcinoma), PC-3M (human prostate adenocarcinoma), HepG2 (human hepatocarcinoma)

Abbreviations used: EGFP, enhanced green fluorescent protein; KHC, kinesin heavy chain; KIF, kinesin superfamily; KLP, kinesin-like protein; NLS, nuclear localization signal; ORF, open reading frame; UTR, untranslated region; Xklp1, Xenopus KLP1.

1 These authors made equal contributions to this study.

2 To whom correspondence should be addressed (e-mail wkim@madang.ajou.ac.kr). 
and Chang (human liver, HeLa marker) cells were grown in Dulbecco's modified Eagle's medium supplemented with $10 \%$ (v/v) fetal bovine serum. Jurkat (human acute T-cell leukaemia) and SH-SY5Y (human neuroblastoma) cells were grown in RPMI 1640 medium supplemented with $10 \%$ (v/v) fetal bovine serum.

\section{Primers}

Primer sequences used for removal or insertion of specific oligopeptides were as follows: 5'-AAAGAAAAAAAGAGGTCTGGCAAACTCCGGAGGCGTACA-3' for removal of ENPPP (where single-letter amino-acid notation has been used); 5' -TCTGGGGAGAATCCACCTCCTACATTCTCCCTTACTGAAGTG-3' for removal of KLRRR; 5'-CCTCCTAAACTCCGGAGGCTGGAAGTGCGTGGTCAAGTTTCG-3' for removal of TFSLT; 5'-GTCGAATTCCCACCTCCTAAACTCCGGAGGCGTGAATTCGTC-3' for insertion of PPPKLRRR; 5'-GTCGAATTCCCTCCTAAACTCCGGAGGCGTGAATTCGTC-3' for insertion of PPKLRRR; 5'-GTCGAATTCCCTAAACTCCGGAGGCGTGAATTCGTC-3' for insertion of PKLRRR; 5'-GTCGAATTCCCTAAACTCCGGAGGGAATTCGTC-3' for insertion of PKLRR; and 5'GTCGAATTCAAACTCCGGAGGCGTGAATTCGTC-3' for insertion of KLRR.

\section{Antibody preparation}

The C-terminal portion of human KIF4 (amino acids 858-1232) was amplified by PCR using the human KIF4 ORF clone as a template. PCR products were cloned into the Easy-T vector (Promega, Madison, WI, U.S.A.), and the DNA insert was excised by EcoRI/XhoI double digestion and cloned into the histidine tag vector, pET30b (Novagen, Madison, WI, U.S.A.), or the glutathione S-transferase vector, pGEX-4T-3 (Pharmacia, Piscataway, NJ, U.S.A.). Resulting plasmids were subsequently transformed into Escherichia coli BL21 (DE3). Fusion proteins were expressed and purified according to the procedures recommended by the manufacturers. Histidine tag fusion proteins were then used as the antigen for the production of anti-KIF4 serum in mice. Anti-KIF4 antibodies were further affinity-purified from antiserum using glutathione S-transferase fusion protein-bound beads as described previously [21].

\section{Western-blot analysis}

Samples prepared from whole cells, or subcellular or chomatographic fractions were resolved by SDS/PAGE and elecrotransferred on to nitrocellulose membranes. Following incubation with blocking buffer [5\% (w/v) non-fat milk powder and $0.1 \%$ Tween 20 in Tris-buffered saline] and then with blocking buffer containing purified anti-KIF4 antibodies, the membranes were washed and then incubated with blocking buffer containing diluted horseradish peroxidase-conjugated anti-mouse IgG antibodies (Amersham, Boston, MA, U.S.A.). The reactive band was visualized using $\mathrm{ECL}^{\circledR}$ (Amersham).

\section{Immunocytochemistry}

A coverslip with cells attached was washed, fixed and then permeabilized with $0.15 \%(\mathrm{v} / \mathrm{v})$ Triton X-100 in $1 \times$ PBS. After brief washes, the coverslip was incubated with blocking solution $[2 \%(\mathrm{w} / \mathrm{v}) \mathrm{BSA}$ in $1 \times \mathrm{PBS}]$ and subsequently with affinitypurified anti-KIF4 antibodies and Cy3-conjugated goat antimouse IgG (Jackson Immunoresearch Laboratories, West Grove, PA, U.S.A.). Fluorescence was observed with a fluorescence microscope or with a confocal laser scanning microscope (LSM510; Zeiss, Thornwood, NJ, U.S.A.).

\section{Subcellular fractionation}

HeLa cells were harvested and washed. The pellet was resus-

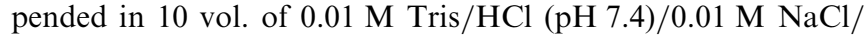
$1.5 \mathrm{mM} \mathrm{MgCl}_{2}$, and the suspension was homogenized with a Dounce homogenizer. When homogenization was complete, as checked with a phase-contrast microscope, the homogenate was centrifuged at $1000 \mathrm{~g}$ for $3 \mathrm{~min}$ at $4{ }^{\circ} \mathrm{C}$. The supernatant and the pellet, representing cytoplasmic and nuclear fractions respectively, were boiled in sample buffer along with whole cells, and subjected to Western-blot analysis.

For DNase I treatment, HeLa cells were harvested by centrifugation and washed. Nuclei were isolated by mild homogenization followed by centrifugation at $500 \mathrm{~g}$. The pellet was resuspended in 2 vol. of $25 \mathrm{mM}$ Hepes ( $\mathrm{pH} 7.9$ ) $/ 0.75 \%$ Triton $\mathrm{X}-100 / 10 \mathrm{mM}$ EDTA/2 $\mathrm{mM} \mathrm{MgCl}_{2}$. The resuspended cell pellet was placed on ice for $10 \mathrm{~min}$, and then centrifuged at $12000 \mathrm{~g}$ for $10 \mathrm{~min}$ at $4{ }^{\circ} \mathrm{C}$. The recovered supernatant represented soluble nucleoplasmic protein. The remaining pellet was washed with DNase I buffer [ $40 \mathrm{mM}$ Tris/ $\mathrm{HCl}(\mathrm{pH} 7.9), 10 \mathrm{mM}$ $\mathrm{NaCl}$ and $6 \mathrm{mM} \mathrm{MgCl}_{2}$ ], resuspended in 2 vol. of DNase I buffer containing 1 unit of DNase $\mathrm{I} / \mu \mathrm{l}$, incubated at $37^{\circ} \mathrm{C}$ for $10 \mathrm{~min}$, and centrifuged. The resulting supernatant and pellet represented DNA-binding proteins and nuclear-matrix-binding proteins respectively.

\section{Construction of deletion/insertion mutants}

All of the deletions and insertions below were confirmed by DNA sequencing. For rough localization of the NLS in the KIF4 ORF, several deletion mutants were constructed (see Figure 3A). First, the insert containing KIF4 ORF was cloned into the SalI/ApaI sites of the pEGFP-C2 vector (where EGFP corresponds to enhanced green fluorescent protein; ClonTech Laboratories, Palo Alto, CA, U.S.A.), yielding C2/KIF4 ORF that was used for the construction of KIF4 $41-5$. KIF $4 \Delta 1$ and KIF $4 \Delta 2$ were produced by removing the internal Bam HI and $E c o$ RI fragments from C2/KIF4 ORF respectively. KIF4 $\Delta 3$ was generated by removing the $B a m \mathrm{HI}$ fragment from $\mathrm{KIF} 4 \Delta 2$, and $\mathrm{KIF} 4 \Delta 4$ and KIF4 45 were obtained by ligation of partially digested C2/KIF4 ORF with BamHI. Secondly, the EcoRI fragment of $2460 \mathrm{bp}$ (amino acids 7-827) was cloned into the pEGFP-C3 vector (ClonTech Laboratories) to maintain the reading frame, yielding KIF4 $\Delta 6$. Partial digestion of KIF4 $\Delta 6$ with $P s t \mathrm{I}$ and re-ligation gave rise to KIF4 47 and KIF4 $\Delta 8$. For acquisition of KIF4 $\Delta 9$, the PstI fragment of $252 \mathrm{bp}$ (amino acids 742-826) was ligated to the largest fragment of KIF4 $\Delta 6$ completely digested with PstI.

To confirm the location of the NLS, additional deletion mutants in which the NLS was abolished were constructed. Thus the C2/KIF4 ORF DNA was cut with $B s p E I$, the site of which is located in the middle of the third NLS candidate (see Figure 3A), in combination with BamHI or SalI, filled-in with Klenow

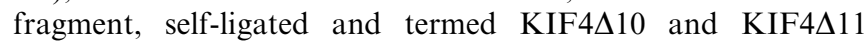
respectively.

For identification of the NLS at the amino acid level, particular oligopeptides were removed from C2/KIF4 ORF or inserted into the $\mathrm{pEGFP-C2}$ vector. Removal was carried out using a sitedirected mutagenesis kit from Stratagene (La Jolla, CA, U.S.A.). With each reaction mixture containing $\mathrm{C} 2 / \mathrm{KIF} 4 \mathrm{ORF}$ DNA (10 ng), sense and antisense primers (125 ng of each) and $P f u$ DNA polymerase (0.5 unit), amplification was performed (1 cycle of $95^{\circ} \mathrm{C}$ for $30 \mathrm{~s}$, and 18 cycles of $95^{\circ} \mathrm{C}$ for $30 \mathrm{~s}, 55^{\circ} \mathrm{C}$ for 
$1 \mathrm{~min}$ and $68^{\circ} \mathrm{C}$ for $22 \mathrm{~min}$ ). Following digestion of parental supercoiled KIF4 ORF DNA with DpnI and transformation into XL1-Blue, deletion was confirmed by DNA sequencing. For insertions, sense and antisense primers were incubated at $55^{\circ} \mathrm{C}$ for $30 \mathrm{~min}$ to form double-stranded oligonucleotides, cut with $E c o$ RI and ligated to the pEGFP-C2 vector digested with EcoRI.

\section{Transient transfection}

To introduce plasmids into HeLa cells, electroporation was performed as recommended by the manufacturer (Bio-Rad, Hercules, CA, U.S.A.). Fluorescence was examined $48 \mathrm{~h}$ after transfection.

\section{RESULTS}

\section{Human KIF4 is a nuclear protein}

Computer analysis of the human KIF4 amino acid sequence indicated that this protein might exist in the nucleus, with a probability of $82.6 \%$. To confirm this possibility by immunocytochemistry and Western-blot analysis, human KIF4-specific antibodies were first prepared. Since the stalk/tail domain of kinesin motors is divergent among KIF members, as mentioned above, the C-terminal portion of human KIF4 (amino acids 858-1232; Figure 1A) was amplified and cloned into the pET expression vector. Overexpressed hexahistidine-tagged human
$\mathrm{KIF}_{858-1232}$ of approx. $51 \mathrm{kDa}$ was purified using $\mathrm{Ni}^{2+}$-bead column chomatography (Figure 1B), dialysed in PBS and injected into Balb/c mouse for antibody production. The specificity of this antibody was determined by Western-blot analysis of HeLa cells. As shown in Figure 1(C), a single band of $140 \mathrm{kDa}$ was easily detected, indicating that the anti-(human KIF4) antibodies detected authentic human KIF4 protein. When its expression in various human cell lines was examined, all samples expressed detectable levels of KIF4 with no cell type-specificity, even though a relatively low level of KIF4 was expressed in HepG2 cells (Figure 1D).

\section{Subnuclear localization of human KIF4}

Next, to determine localization of the human KIF4 protein, immunostaining of HeLa cells was carried out, and it was found that the human KIF4 protein was localized mainly in the nucleus, with a small amount in the cytoplasm (Figure 2A). It should also be noted that KIF4 was not present in the nucleolus.

To confirm the above bimodal localization observed by immunostaining, subcellular fractionation of HeLa cells was performed. Therefore, after disrupting the cells with mild homogenization, the nuclear fraction was separated from the cytosolic fraction by centrifugation, and both fractions were then subjected to Western-blot analysis. As shown in Figure 2(B), both fractions

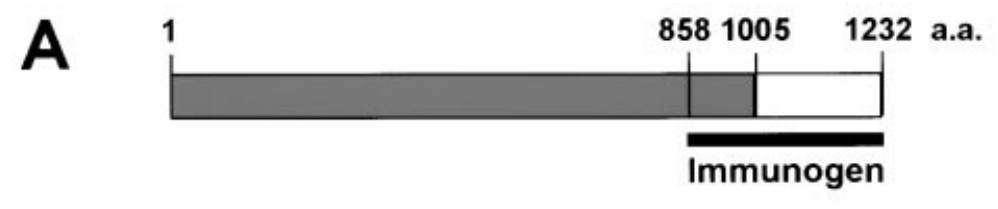

B
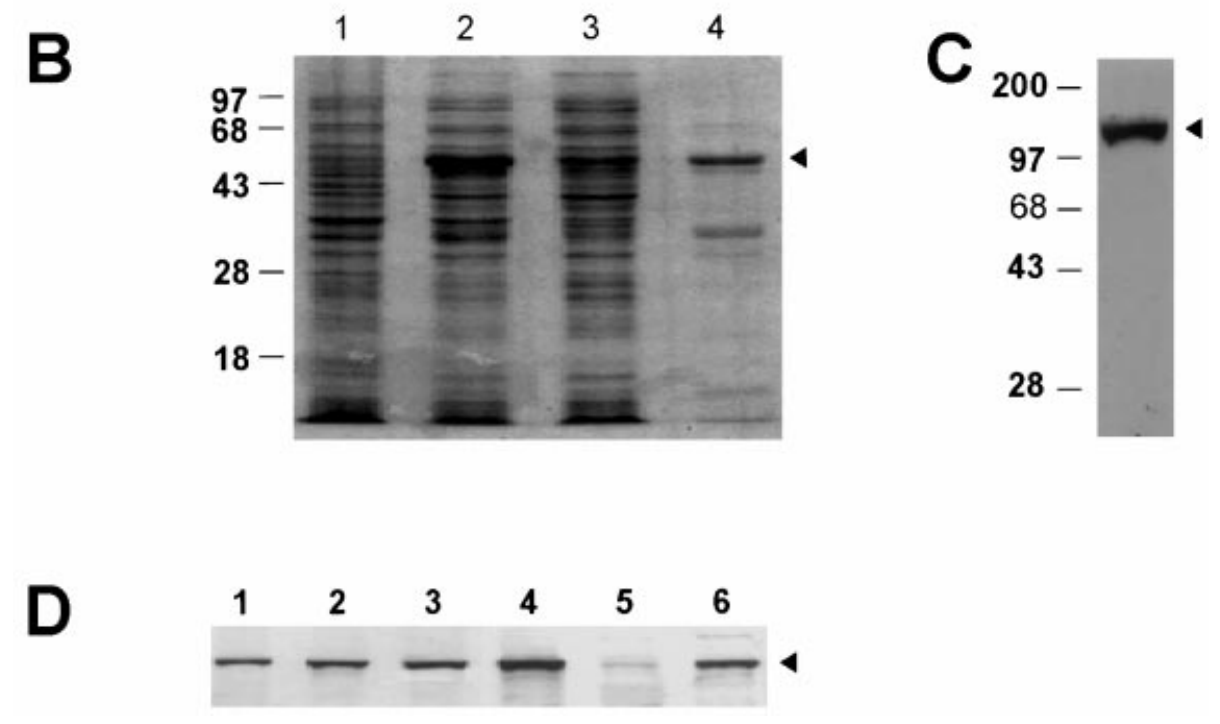

\section{Figure 1 Preparation of the anti-KIF4 antibody}

(A) A schematic illustration of the entire human KIF4 ORF, including the C-terminal portion that was used to prepare antibodies. The shaded box represents the motor and stalk domains, and the open box represents the binding domain. Abbreviation: a.a., amino acid. (B) Expression of the hexahistidine-fused C-terminal portion. Expressed protein was analysed on an SDS/12\% (w/v) polyacrylamide gel, which was subsequently stained with Coomassie Brilliant Blue R-250. The arrowhead indicates the band used as the immunogen. Lane 1 , uninduced bacterial lysate; lane 2 , induced bacterial lysate; lane 3, sonicated bacterial supernatant; lane 4, purified hexahistidine-fused KIF4 (858-1232) protein (purified using an Ni ${ }^{2+}$ column). Western-blot analysis of KIF4 in HeLa cells (C) and various human cell lines (D). Total extract (30 $\mu \mathrm{g})$ was run on an SDS/6.5\% (w/v) polyacrylamide gel. After transferring on to nitrocellulose, Western-blot analysis was performed. The arrowhead indicates human KIF4 (140 kDa). Lanes in (D): 1, HeLa cells; 2, Chang cells; 3, PC-3M cells; 4, Jurkat cells; 5, HepG2 cells; and 6, SH-SY5Y cells. 

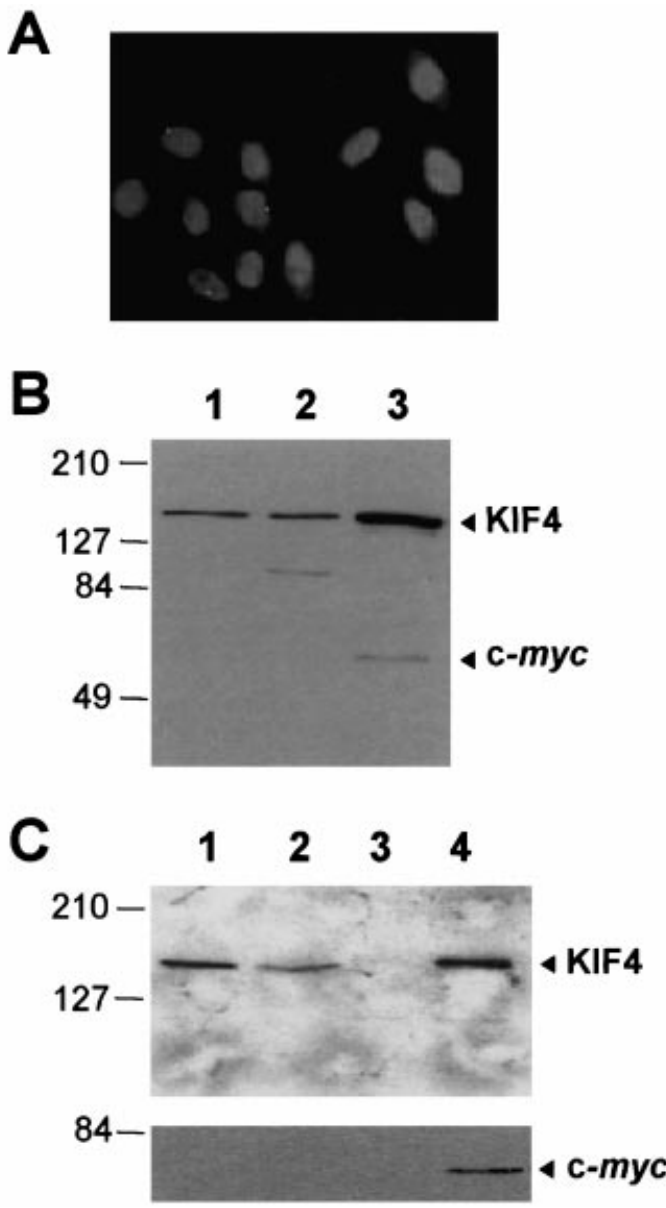

Figure 2 Nuclear localization of KIF4

(A) Immunostaining of HeLa cells at interphase with anti-(human KIF4) antibodies. (B) Subcellular fractionation of HeLa cells. Proteins $(40 \mu \mathrm{g})$ were run on an SDS/8\% (w/v) polyacrylamide gel. Lane 1, total cell extract; lane 2, cytosol; lane 3, nucleoplasm. c-Myc protein was used as a control for a nuclear protein. (C) Subnuclear fractionation. After fractionation, each fraction was adjusted to $260 \mu$ l and one-tenth of this volume was analysed. Lane 1, total cell extract of HeLa cells ( $40 \mu \mathrm{g}$ ); lane 2, nucleoplasmic fraction; lane 3, DNase I-soluble fraction; lane 4, nuclear matrix fraction. c-Myc protein was used as a control for a nuclear matrix binding protein

contained KIF4, and the ratio of their intensity was in good accord with the above immunostaining result.

Information on the subnuclear localization of human KIF4 is a prerequisite for a detailed study on its function(s). In general, nuclear proteins can be fractionated into three subfractions, based on their locations. Soluble nuclear proteins present in the nucleoplasm are easily extractable by treatment with mild detergent, and a nuclear pellet resulting from centrifugation of detergent-treated nuclei is composed of DNA, DNA binding proteins and nuclear matrix. When resuspended post-detergent pellet is extensively treated with DNase I, followed by centrifugation, the DNA-binding proteins remain in the supernatant and proteins associated with the nuclear matrix reside in the resulting pellet. Therefore HeLa nuclei prepared after Dounce homogenization were fractionated into the above three subfractions. Western-blot analysis showed that the nuclear KIF4 was predominantly confined to the nuclear matrix (Figure 2C). As expected, c-Myc, known to be associated with the nuclear matrix [22,23], co-localized with KIF4.
Table 1 Sequence comparison of four NLS candidates in KIF4 members

H, human (Homo sapiens) [20]; M, mouse (Mus musculus) [17]; C, chick (Gallus gallus) [24]; $\mathrm{F}$, frog (Xenopus laevis) [25].

\begin{tabular}{|c|c|}
\hline Candidate number & Sequence \\
\hline 1 & 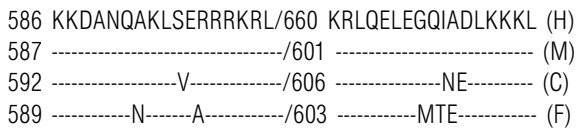 \\
\hline$\|$ & 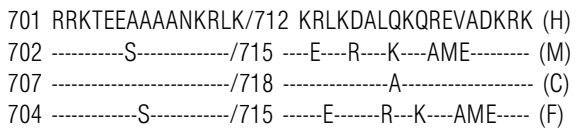 \\
\hline III & $\begin{array}{l}790 \text { NPPPKLRRRT }(\mathrm{H}) \\
791 \text {-----L---------- }(\mathrm{M}) \\
796 \text {----S---------- (C) } \\
793 \text { Rl---T--|-------- (F) }\end{array}$ \\
\hline IV & $\begin{array}{l}1207 \text { GKKKRA (H) } \\
1206 \text {-------- }(\mathrm{M}) \\
1200 \mathrm{~V} \text {------M (C) } \\
1202 \text { T-----VL (F) }\end{array}$ \\
\hline
\end{tabular}

\section{Determination of the NLS of human KIF4}

Considering the above results, we concluded that human KIF4 was a nuclear protein. This meant the possibility of an NLS in human KIF4. Computer analysis of the human KIF4 amino acid sequence indicated four putative NLS candidates, all of which are well conserved among KIF4 members of higher eukaryotes (Table 1). Consequently, the determination of the NLS was initiated by cloning the entire KIF4 ORF into the pEGFP-C2 vector, yielding KIF4 ORF as a positive control. From that vector, the NLS-deficient vector containing only a short Nterminal portion (KIF $\Delta 1$ ) was generated. Transient expression of fused fluorescence products for KIF4 ORF was observed in the nucleus of HeLa cells and for KIF $\Delta 1$ in the cytoplasm, as expected (Figure 3B). Using KIF4 ORF as a parental vector, an additional four deletion mutants (KIF $\Delta 2-5)$ were constructed, and only KIF $\Delta 5$ harbouring the first three candidates (see Figure 3A) was found to direct KIF4 to the nucleus, eliminating the last candidate.

To resolve the above three candidates further, the EcoRI fragment covering amino acids 7-827 was cloned into pEGFP$\mathrm{C} 3$ to maintain the reading frame, yielding KIF4 $\Delta 6$, which also expressed fluorescent nuclear protein. From this plasmid, three additional deletion mutants (KIF $\Delta 7-9$ ) were constructed. Of these, KIF $\Delta 9$ exhibited nuclear localization as KIF $\Delta 6$ did, thus narrowing down the NLS to the third candidate.

The predicted NLS sequence in the third candidate is PPPKLRRR. To our delight, the BspEI site in the middle of PPPKLRRR enabled us to directly confirm the real NLS. Complete digestion of KIF4 ORF by combination of BamHI or EcoRI, filling-in with Klenow fragment, and self-ligation yielded KIF4 410 ending with PPP and KIF4 411 starting with KLRRR respectively. When transfected, both expressed a protein that was restricted to the cytoplasm, thus indicating the NLS of KIF4 protein should reside around the sequence of PPPKLRRR. The results obtained from deletion mutants are summarized in Figure 3(C).

To identify the NLS at the amino acid level, particular oligopeptides were removed from $\mathrm{C} 2 / \mathrm{KIF} 4 \mathrm{ORF}$ or inserted into the EGFP-C2 vector. When ENPPP, KLRRR or TFSLT were removed, a nuclear fluorescent protein was encoded only by 
A

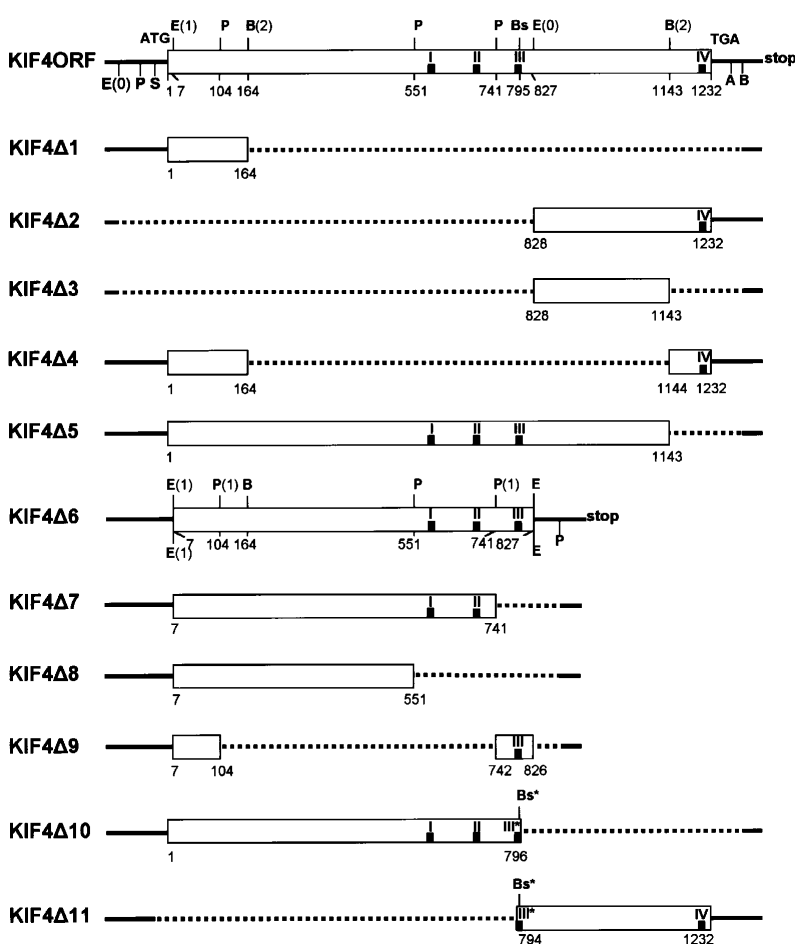

B
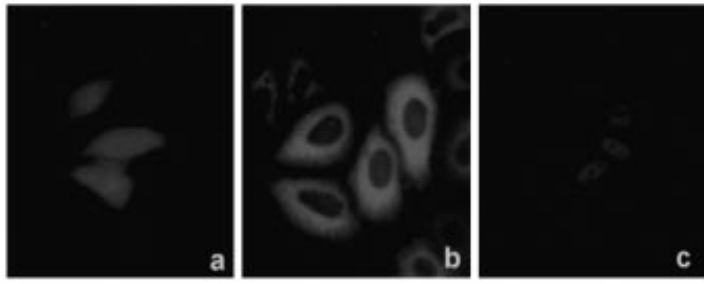

C

\begin{tabular}{|c|c|c|c|c|}
\hline Clone & Vector (EGEP) & Deletions (a.a.) & Nuclear & localization \\
\hline KIP 4ORF & $\mathrm{C} 2$ & None & & Yes \\
\hline $\mathrm{KIF} 4 \Delta 1$ & $\mathrm{C} 2$ & $165-1232$ & & No \\
\hline KIF $4 \Delta 2$ & c2 & $1-826$ & & No \\
\hline $\operatorname{KIF} 4 \Delta 3$ & C2 & $1-826 ; 1144-1232$ & & No \\
\hline KIF $4 \Delta 4$ & $\mathrm{c} 2$ & $165-1143$ & & No \\
\hline KIF $4 \Delta 5$ & C2 2 & $1144-1232$ & & Yes \\
\hline KIF $4 \Delta 6$ & $\mathrm{C} 3$ & $1-6 ; 828-1232$ & & Yes \\
\hline KIF $4 \Delta 7$ & c3 3 & $1-6 ; 742-1232$ & & No \\
\hline $\mathrm{KIF} 4 \Delta 8$ & $\mathrm{c} 3$ & $1-6 ; 552-1232$ & & No \\
\hline KIF $\triangle \Delta 9$ & c3 3 & $\begin{array}{l}1-6 ; 105-741 ; \\
828-1232\end{array}$ & & Yes \\
\hline KIFA $A 10$ & $\mathrm{C} 2$ & $797-1232$ & & No \\
\hline $\operatorname{KIF} 4 \Delta 11$ & $\mathrm{C} 2$ & $1-793$ & & No \\
\hline
\end{tabular}

\section{Figure 3 Localization of NLS}

(A) Schematic illustration of KIF4 deletants. The entire and partial ORFs of KIF4 were fused to the EGFP vector (thick line). Deleted regions are indicated by broken lines. Bold letters above the boxes and below the thick lines are restriction-enzyme sites of the KIF4 ORF and pEGFP vector respectively. Small black boxes show the locations of predicted NLS candidates numbered in roman numerals (see Table 2). Nullified BspEl sites and NLSs created by Klenow treatment (see the Experimental section) are marked with asterisks. The numbers in parentheses indicate the frame of the enzyme sites. A, Apal; B, BamHI; Bs, BspEl; E, EcoRl; P, Pstl; S, Sall. (B) Fluorescence detection. Green fluorescence in HeLa cells transfected with the EGFP-C2 (a), KIF4 1 (b) or KIF4 $\Delta 5$ (c) was examined $48 \mathrm{~h}$ after transfection. (C) Summary of nuclear localization of KIF4 deletions.

Table 2 Determination of the KIF4 NLS at the amino acid level

Parental DNAs were KIF4 ORF for deletion and EGFP for insertion.

\begin{tabular}{lll}
\hline Deletion (D)/insertion (I) & & Nuclear localization \\
\hline SG-----KLRRRTFSLTEV & (D) & No \\
SGENPPP-----TFSLTEV & (D) & No \\
SGENPPPKLRRR-----EV & (D) & Yes \\
---- PPPKLRRR------ & (I) & Yes \\
----- PPKLRRR------ & (I) & Yes \\
------ PKLRRR------ & (I) & Yes \\
$-------K L R R R------$ & (I) & No \\
$------P K L R R-------$ & (I) & No
\end{tabular}

the plasmid with TFSLT deleted (Table 2). Next, we inserted the PPPKLRRR sequence into the EGFP-C2 vector and found that it functioned as an NLS. When several oligopeptides with amino acid shortening one by one from either end of PPPKLRRR were tested, the minimum sequence of the KIF4 NLS was found to be PKLRRR (Table 2).

\section{Chromosomal association of human KIF4}

Biochemical analysis of HeLa cells using affinity-purified human KIF4 antibodies showed that human KIF4 was related to chicken chromokinesin, which is localized in the nucleus [24]. In addition, its primary association with chromosomes has suggested that chicken chromokiensin is likely to function as a microtubulebased mitotic motor. In the present study we determined whether human KIF4 was associated with chromosomes in mitotic cells. Confocal microscopic observation of mitotic HeLa cells stained simultaneously with KIF4-specific antibodies and 4,6-diamidino2-phenylindole revealed that human KIF4 was uniformly associated with chromosomes at all stages of mitosis (Figure 4). Exclusive distribution of KIF4 to chromosomes and its identical images with those of chromosomes suggests that KIF4 might be involved in chromosome movement during mitosis. In this sense, human KIF4 seems to be functionally related to chick chromokinesin.

\section{DISCUSSION}

Kinesin and kinesin-related genes have been identified in various organisms ranging from yeast to humans, comprising a superfamily [1]. They can be divided into two groups; one responsible for the transport of membraneous organelles or vesicles and the other responsible for mitotic or meiotic DNA transport. According to the recent phylogenetic analysis of kinesins (http:// www.blocks.fhcrc.org/ kinesin/), the KIF4 subfamily includes four vertebrate [human and murine KIF4s, chick chomokinesin and Xenopus KLP1(Xklp1)] and four invertebrate (KLP31D and KLP3A of Drosophila, and T01G1 and Y43F4B of Caenorhabditis elegans) kinesins. Of these, only vertebrate members, except for the human one, have been characterized to some 

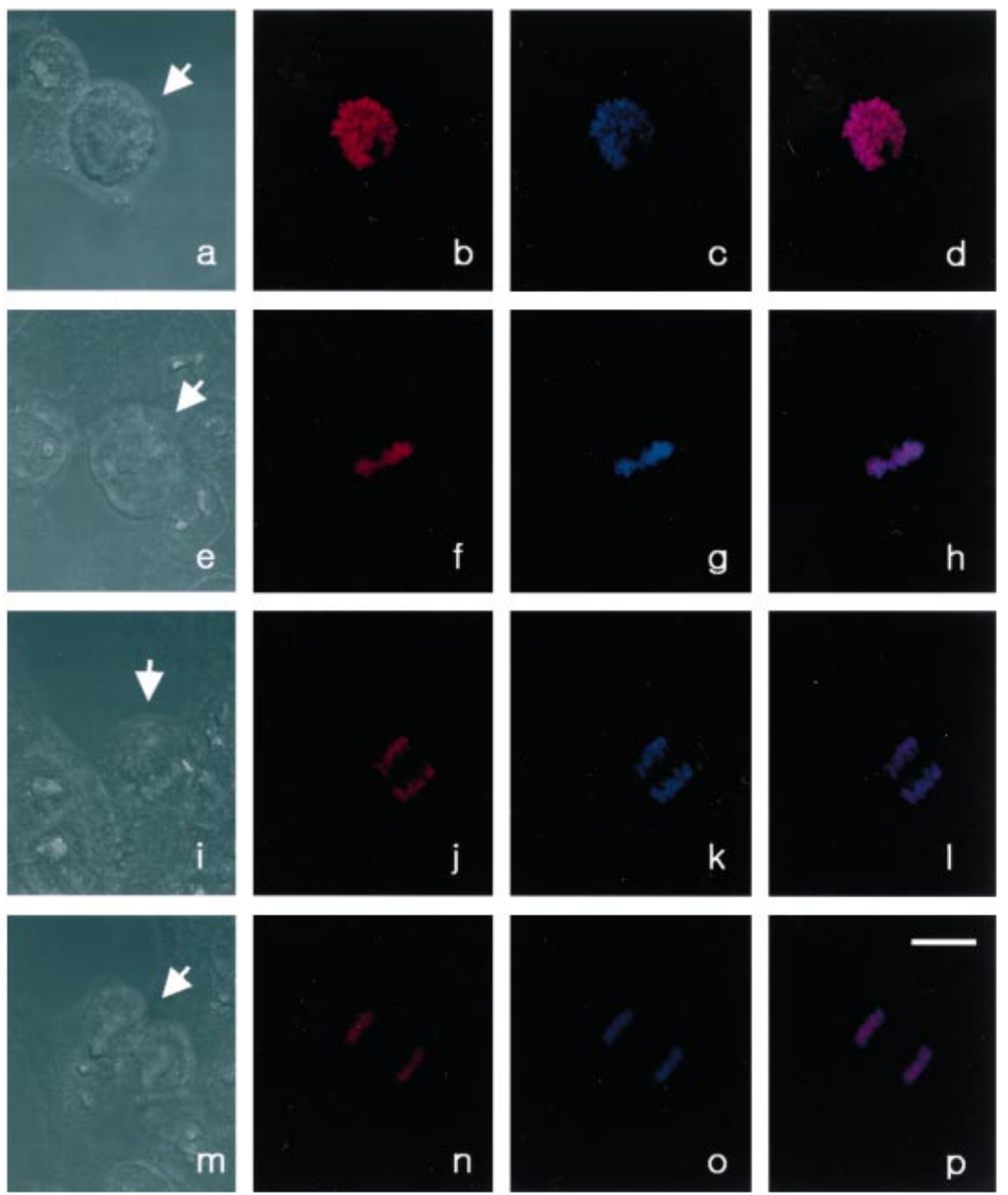

\section{Figure 4 Association of human KIF4 with chromosomes in mitotic cells}

Mitotic HeLa cells were double stained with anti-(human KIF4) antibodies and 4,6-diamidino-2-phenylindole, and were observed with a confocal laser scanning microscope. Arrows indicate cells that were focused for fluorescent images. Cells at prophase $(\mathbf{a}-\mathbf{d})$, metaphase $(\mathbf{e}-\mathbf{h})$, anaphase $(\mathbf{i}-\mathbf{I})$ and telophase $(\mathbf{m}-\mathbf{p})$ were visualized by bright-field fluorescence $(\mathbf{a}, \mathbf{e}, \mathbf{i}$ and $\mathbf{m})$, Cy-3 fluorescence for human KIF4 (b, $\mathbf{f}, \mathbf{j}$ and $\mathbf{n}), 4,6$-diamidino-2-phenylindole fluorescence for DNA (c, $\mathbf{g}, \mathbf{k}$ and $\mathbf{o})$, and a combination of Cy-3 and 4,6-diamidino-2-phenylindole fluorescence (d, $\mathbf{h}, \mathbf{I}$ and $\mathbf{p})$. Bar $=20 \mu \mathrm{m}$.

extent, if not completely. Chick chomokinesin with DNA binding activity is also a nuclear protein and associates with chromosome arms [24], and Xklp1, which is considered to be a nuclear protein, positions mitotic chromosomes and stabilizes bipolar mitotic spindles [25]. Murine KIF4 is found in both the cytoplasm and nucleus, and co-localizes with membraneous organelles in interphase cells and with spindle microtubules in mitotic cells [1]. Recently, it has been shown that KIF4 is involved in the anterograde transport of vesicles containing L1, a cell-adhesion molecule implicated in axonal elongation [26]. One common function of these three KIF4 subfamily members is their association with the mitotic apparatus. Hence, among KIF4 subfamily members, murine KIF4 is the only motor known for vesicle transport. In terms of sequence similarity and subcellular distribution, human KIF4 is most closely related to murine KIF4, and it has been suggested that human KIF4 possesses similar functions to those of murine KIF4. However, in spite of $68 \%$ amino acid similarity between them, the presence of several divergent regions in the binding domain [20] underscores the possibility that human KIF4 is not a functional orthologue of murine KIF4. As an initial step to address the function of human KIF4, we examined its localization and chromosomal association in the present study. Human KIF4 was found to localize to HeLa cell nuclei, and nuclear localization was further supported by fractionation techniques, where it was found that human KIF4 was present in the insoluble nuclear fraction (nuclear matrix). The NLS of human KIF4 was defined by deletion and mutagenesis of EGFP-KIF4 fusion proteins, followed by transfection and localization. In addition, human KIF4 strongly and exclusively associated with chromosomes in mitotic cells. Taking these results together, we conclude that human KIF4 is mostly related to chick chromokinesin and is likely to function as a mitotic motor transporting chromosomes. However, partial, if not predominant, cytoplasmic localization of human KIF4 in interphase cells does not exclude the possibility that it functions as an intracellular membranous organelle-transporting motor 
similar to murine KIF4. Even though a novel function of human KIF4 is not directly addressed, these initial findings that contrast with cytoplasmic murine KIF4 function provide a basis for further functional characterization of human KIF4.

Bimodal localization of proteins makes it difficult to define their subcellular destiny. Usually, a protein containing an NLS and functioning in the nucleus is classified as a nuclear protein, even though proteins such as nuclear factor $\kappa \mathrm{B}$ [27] and cyclindependent kinase 2 [28] are retained temporarily in the cytoplasm by association with a molecule that inhibits nuclear targeting. Equally, a protein that functions in the cytoplasm is a cytoplasmic protein. So is human KIF4 a cytoplasmic or a nuclear protein? Since murine KIF4, which is closely related to human KIF4, is involved in the anterograde transport of a cytoplasmic vesicle $[17,26]$, it is highly likely that human KIF4 carries out a similar function. However, as mentioned above, some divergence in the putative binding domains between murine and human KIF4 proteins alludes to other options. Categorization of human KIF4 should be awaited until its function is elucidated.

Dominant association with the nuclear matrix raises a question as to whether human KIF4 is involved in regulation of gene expression, even though the exact biological function of the nuclear matrix, on which gene expression has been suggested to depend to some degree, remains uncertain ([29] and references therein). Since transcription is modulated by a multi-protein complex, involvement of human KIF4 in that process could be inferred by identification of KIF4-interacting regulatory proteins. In addition to the tail domain that is considered to bind to cargoes, the coiled-coil stalk domain possibly provides the basis for interaction with other protein(s). In fact, it was shown that interaction between the Kar3 motor protein from yeast and its associated protein Cik 1 is mediated by their respective coiled-coil domains [30].

Nuclear import of proteins involves recognition of NLS by members of the importin family of proteins, which mediate crossing across the nuclear envelope. NLSs can be divided into four groups [31]. Among 'simple', 'bipartite' or 'split', 'nonpositive' and 'highly basic' NLSs, the first two candidates are bipartite NLSs and the rest are simple NLSs in the case of human KIF4 (see Table 1). All these sequences are relatively well conserved among KIF4 members. However, only the third one (PKLRRR) was found to direct nuclear localization of human KIF4. This observation suggests that some surrounding sequences, in addition to the NLS itself, may be necessary for nuclear localization of a particular protein, such as KIF4. Moreover, based on comparison with the known Xenopus nuclear proteins, the predicted NLSs of Xklp1 corresponded to the first two candidates [25], neither of which functioned as an NLS in the present study. These results indicate that NLSs of KIF4 members require a precisely defined sequence. The degree to which each residue of the KIF4 NLS (PKLRRR) contributed to nuclear localization was not investigated in the present study. As shown in Table 1, KL(I)RRR is well conserved among KIF4 members, whereas the proline residue is not. Rather, the preceding proline residue (the second proline of PPP) is conserved. The significance of the non-conserved proline residue (the third proline of PPP), however, should not be underestimated until NLSs of other species are identified.

In summary, it was found that most, if not all, KIF4 was localized in the nucleus and that nuclear KIF4 was predominantly associated with the nuclear matrix. Although the biological significance of cytoplasmic localization and association with the nuclear matrix remains obscure, its exclusive association with the chromosomes at all phases of mitosis suggests that human KIF4 is likely to be a mitotic motor with DNA as its cargo.
When the significance of such non-chromosomal localization is elucidated, the function of human KIF4 will be extended beyond chromosomal association.

We are grateful to Dr Woon Ki Paik and Dr Mahn Joon Ha for helpful discussions and critical reading of the manuscript. We also thank Mr Sungmin Ahn for technical assistance.This work was supported by grant number 2000-1-20900-009-3 from the Basic Research Program of the Korea Science \& Engineering Foundation (to W. K.), and in part by the Brain Korea 21 Project (to W.C.).

\section{REFERENCES}

1 Rieder, C. L. and Salmon, E. D. (1998) The vertebrate cell kinetochore and its roles during mitosis. Trends Cell Biol. 8, 310-318

2 Heald, R. and Walczak, C. E. (1999) Microtubule-based motor function in mitosis. Curr. Opin. Struct. Biol. 9, 268-274

3 Hirokawa, N. (1998) Kinesin and dynein superfamily proteins and the mechanism of organelle transport. Science (Washington, D.C.) 279, 519-526

4 Goldstein, L. S. B. and Yang, Z. (2000) Microtubule-based transport system in neurons: the roles of kinesin and dynein. Annu. Rev. Neurosci. 23, 39-71

5 Hirokawa, N., Noda, Y. and Okada, Y. (1998) Kinesin and dynein superfamily protein in organelle transport. Curr. Opin. Cell Biol. 10, 60-73

6 Vale, R. D., Schnapp, B. J., Reese, T. S. and Sheetz, M. P. (1985) Movement of organelles along filaments dissociated from the axoplasm of the squid giant axon. Cell (Cambridge, Mass.) 40, 449-454

7 Vale, R. D., Schnapp, B. J., Reese, T. S. and Sheetz, M. P. (1985) Organelle, bead, and microtubule translocations promoted by soluble factors from the squid giant axon. Cell (Cambridge, Mass.) 40, 559-569

8 Brady, S. T. (1985) A novel brain ATPase with properties expected for the fast axonal transport motor. Nature (London) 317, 73-75

9 Bloom, G. S., Wagner, M. C., Pfister, K. K. and Brady, S. T. (1988) Native structure and physical properties of bovine brain kinesin and identification of the ATP binding subunit polypeptide. Biochemistry 27, 3409-3416

10 Kuiznestov, S. A., Vaisberg, E. A., Shanina, N. A., Magvetova, N. N., Chernyak, V. X. and Gelfand, V. I. (1988) The quaternary structure of bovine brain kinesin. EMBO J. 7, 353-356

11 Yang, J. T., Saxton, W. M., Stewart, R. J., Raff, E. C. and Goldstein, L. S. B. (1990) Evidence that the head of kinesin is sufficient for force generation and motility in vitro. Science (Washington, D.C.) 249, 42-47

12 Yang, J. T., Laymon, R. A. and Goldstein, L. S. B. (1989) A three-domain structure of kinesin heavy chain revealed by DNA sequence and microtubule binding analyses. Cell (Cambridge, Mass.) 56, 879-889

13 Kosik, K. S., Orecchio, L. D., Schnapp, B., Inouye, H. and Neve, R. L. (1990) The primary structure and analysis of the squid kinesin heavy chain. J. Biol. Chem. 265 3278-3283

14 Goldstein, L. S. B. (1991) The kinesin superfamily: tails of functional redundancy. Trends Cell Biol. 1, 93-98

15 Nakagawa, T., Tanaka, Y., Matsuoka, E., Kondo, S., Okada, Y., Noda, Y., Kanai, Y. and Hirokawa, N. (1997) Identification and classification of 16 new kinesin superfamily (KIF) proteins in mouse genome. Proc. Natl. Acad. Sci. U.S.A. 94, 9654-9659

16 Aizawa, H., Sekine, Y., Takemura, R., Zhang, Z., Nangaku, M. and Hirokawa, N (1992) Kinesin family in murine central nervous system. J. Cell Biol. 119, 1287-1296

17 Sekine, Y., Okada, Y., Noda, Y., Kondo, S., Aizawa, H., Takemura, R. and Hirokawa, N. (1994) A novel microtubule-based motor protein (KIF-4) for organelle transports, whose expression is regulated developmentally. J. Cell Biol. 127, 187-201

18 Kim, W., Tang, Y., Okada, Y., Torrey, T. A., Chattopadhyay, S. K., Pfleiderer, M. Falkner, F. G., Dorner, F., Choi, W., Hirokawa, N. and Morse, III, H. C. (1998) Binding of murine leukemia virus Gag polyproteins to KIF4, a microtubule-based motor protein. J. Virol. 72, 6898-6901

19 Tang, Y., Winkler, U., Freed, E. O., Torrey, T. A., Kim, W., Li, H., Goff, S. P. and Morse, III, H. C. (1999) Cellular motor protein KIF-4 associates with retroviral Gag. J. Virol. 73, 10508-10513

20 Oh, S., Han, H., Torrey, T. A., Shin, H., Choi, W., Lee, Y. M., Morse, III, H. C. and Kim, W. (2000) Identification of the human homologue of mouse KIF4, a kinesin superfamily motor protein. Biochim. Biophys. Acta 1493, 219-224

21 Olmsted, L. B. (1981) Affinity purification of antibodies from diazotized paper blots of heterogeneous protein samples. J. Biol. Chem. 256, 11955-11957

22 Persson, H. and Leder, P. (1984) Nuclear localization and DNA binding properties of a protein expressed by human c-myc oncogene. Science (Washington, D.C.) 225, $718-721$

23 Eisenman, R. N., Tachibana, C. Y., Abrams, H. D. and Hann, S. R. (1985) V-myc- and c-myc-encoded proteins are assoicated with the nuclear matrix. Mol. Cell. Biol. $\mathbf{5}$, 114-126 
24 Wang, S.-Z. and Adler, R. (1995) Chromokinesin: a DNA-binding, kinesin-like nuclear protein. J. Cell Biol. 128, 761-768

25 Vernos, I., Raats, J., Hirano, T., Heasman, J., Karsenti, E. and Wylie, C. (1995) Xklp1, a chomosomal Xenopus kinesin-like protein essential for spindle organization and chomosome positioning. Cell (Cambridge, Mass.) 81, 117-127

26 Peretti, D., Peris, L., Rosso, S., Quiroga, S. and Caceres, A. (2000) Evidence for the involvement of KIF4 in the anterograde transport of L1-containing vesicles. J. Cell Biol. 149, 141-152

27 Baeuerle, P. A. and Baltimore, D. (1988) I kappa B: a specific inhibitor of the NFkappa B transcription factor. Science (Washington, D.C.) 242, 540-546
28 Wang, G., Miskimins, R. and Miskimins, W. K. (1999) The cyclin-dependent kinase inhibitor p27Kip1 is localized to the cytosol in Swiss/3T3 cells. Oncogene 16, 5024-5010

29 Pederson, T. (2000) Half a century of "the nuclear matrix". Mol. Biol. Cell 11 799-805

30 Barrett, J. G., Manning, B. D. and Snyder, M. (2000) The Kar3p kinesin-related protein forms a novel heterodimeric structure with its associated protein Cik1p. Mol. Biol. Cell 11, 2373-2385

31 Boulikas, T. (1993) Nuclear localization signals (NLS). Crit. Rev. Eukaryotic Gene Expression 3, 193-227

Received 24 July 2001/10 September 2001; accepted 27 September 2001 